\title{
Insegurança alimentar e excesso de peso em escolares do primeiro ano do Ensino Fundamental da rede municipal de São Leopoldo, Rio Grande do Sul, Brasil
}

\author{
Food insecurity and overweight in first grade \\ students in the municipal school system in São \\ Leopoldo, Rio Grande do Sul State, Brazil
}

\section{Inseguridad alimentaria y sobrepeso en niños matriculados en el primer año de la enseñanza primaria en las escuelas municipales de la ciudad de São Leopoldo, Río Grande do Sul, Brasil}

Keli Vicenzi 1

Ruth Liane Henn 1

Ana Paula Weber 1

Vanessa Backes 1

Vera Maria Vieira Paniz 1

Talita Donatti 1

Maria Teresa Anselmo Olinto 1

I Universidade do Vale do
Rio dos Sinos, São Leopoldo,
Brasil.
Correspondência
R. L. Henn
Centro de Ciências da Saúde,
Universidade do Vale do
Rio dos Sinos.
Av. Unisinos 950,
São Leopoldo, RS
93022-000, Brasil.
ruthenn@unisinos.br

Abstract

This cross-sectional school-based study in São Leopoldo, Rio Grande do Sul State, Brazil, evaluated the association between food insecurity and overweight in first grade students in the municipal elementary school system. A total of 2,369 students were invited to participate, of whom 847 were examined, and of these, 782 had data available on weight and height. Dietary data were obtained from a parent or guardian. Food insecurity was measured by the Brazilian Food Insecurity Scale (EBIA). Data on weight and height were provided by the Nutrition Service of the Municipal Department of Education. Prevalence rates for overweight and food insecurity were $38.1 \%$ and $45.1 \%$, respectively. After controlling for potential confounders, children with food insecurity had 22\% lower odds of overweight. Notwithstanding the inverse association between the exposure and outcome, this sample showed high rates of food insecurity and overweight, revealing a complex relationship and indicating that further research is needed to understand it. Robust public policies are critical for addressing these conditions.

Pediatric Obesity; Overweight; Students; Food Security

\section{Resumo}

Estudo transversal, de base escolar, realizado em São Leopoldo, Rio Grande do Sul, Brasil, com o objetivo de avaliar a associação entre insegurança alimentar e excesso de peso em escolares do $1^{\circ}$ ano do Ensino Fundamental das escolas municipais. Dois mil, trezentos e sessenta e nove escolares foram convidados a participar, 847 foram investigados e destes, 782 tinham dados de peso e altura. Os dados foram obtidos com as mães/ responsáveis. Insegurança alimentar foi medida com a Escala Brasileira de Insegurança Alimentar (EBIA). Peso e altura foram obtidos com o Serviço de Nutrição da Secretaria Municipal de Educação. As prevalências de excesso de peso e insegurança alimentar foram, respectivamente, $38,1 \%$ e 45,1\%. Após ajuste para fatores de confusão, escolares com insegurança alimentar apresentaram probabilidade $22 \%$ menor de ter excesso de peso quando comparados com aqueles sem insegurança alimentar. Este estudo identificou elevadas prevalências de insegurança alimentar e excesso de peso, com associação inversa entre estas variáveis. Por um lado, esses resultados revelam a complexidade dessa relação, o que exige mais estudos para compreendê-la, e, por outro, mostra a necessidade de políticas públicas robustas para enfrentar essas condições.

Obesidade Pediátrica; Sobrepeso; Estudantes; Segurança Alimentar e Nutricional 


\section{Introdução}

A obesidade é uma doença crônica não transmissível, e sua prevalência tem apresentado aumento expressivo nas últimas décadas, em toda população mundial, independentemente do estágio do ciclo da vida 1 . Tal situação vem sendo classificada pela Organização Mundial da Saúde (OMS) como a epidemia do século XXI 2. De Onis et al. 3 identificaram 43 milhões de crianças (35 milhões nos países em desenvolvimento) com sobrepeso ou obesidade e 92 milhões em risco de desenvolver estas condições. No Brasil, segundo dados da Pesquisa de Orçamentos Familiares (POF) de 2008-2009, 34\% das crianças com idades entre cinco e nove anos apresentavam excesso de peso, sendo que $14 \%$ delas encontravam-se obesas 4.

Considerada uma condição de desenvolvimento multifatorial, a obesidade apresenta dificuldades no conhecimento pleno de sua etiologia, porém, é consenso na literatura que a gênese desta condição envolve condições biológicas, comportamentais e ambientais ${ }^{5}$. Assim, características no início da vida, como peso de nascimento ${ }^{6}$ e aleitamento materno 7 ; padrões alimentares 8 e de atividade física ${ }^{9}$; influência do ambiente familiar (obesidade dos pais) e condições socioeconômicas da família 8 são fatores que têm sido implicados na ocorrência da obesidade. Mais recentemente, a insegurança alimentar, que ocorre quando o acesso regular e permanente a alimentos de qualidade, em quantidade suficiente, não é garantido 10, também tem sido considerada um fator de risco para obesidade em crianças 11,12. Entretanto, a relação entre insegurança alimentar e obesidade apresenta-se controversa, com estudos mostrando uma associação positiva 11,12 , outros não encontrando nenhuma associação 13,14,15,16, e alguns mostrando risco reduzido de obesidade na presença da insegurança alimentar 17,18.

Considerando que 17,7 milhões de domicílios brasileiros (30,2\%) apresentam algum grau de insegurança alimentar (18,7\% - insegurança alimentar leve; 6,5\% - insegurança alimentar moderada; $5,8 \%$ - insegurança alimentar grave) 19 e que um terço das crianças de cinco a nove anos está com excesso de peso ${ }^{4}$, o presente estudo teve por objetivo verificar a associação entre insegurança alimentar e excesso de peso em escolares do primeiro ano do ensino fundamental das escolas municipais de São Leopoldo, Rio Grande do Sul, Brasil.

\section{Metodologia}

O presente trabalho integra o projeto adesão aos 10 Passos da Alimentação Saudável para Crianças 20 entre escolares do 1o ano das escolas municipais de Ensino Fundamental de São Leopoldo, um estudo transversal, de base escolar, cujo objetivo foi avaliar a frequência de realização de cada passo e fatores associados. São Leopoldo está situada na Região do Vale do Rio dos Sinos e integra a Região Metropolitana de Porto Alegre, distando $34 \mathrm{~km}$ da capital do estado. Em 2011, contava com 35 escolas municipais de Ensino Fundamental, com 2.369 escolares matriculados no 1o ano. No início do ano letivo, o projeto foi apresentado às equipes diretivas das escolas, bem como aos pais/responsáveis, por meio de reuniões ou de uma carta de apresentação. Todos os escolares matriculados no 1o ano foram convidados a participar do estudo, que teve início em maio de 2011. As entrevistas foram agendadas para serem realizadas na escola, contudo, devido ao baixo número de comparecimentos, os entrevistadores passaram a realizá-las nos domicílios. Os endereços dos escolares foram obtidos junto à Secretaria Municipal de Educação. A coleta de dados foi encerrada no final do ano letivo. Aqueles que apresentavam alguma deficiência física que impedisse a tomada de medidas antropométricas, ou realizavam dietas para condições especiais, foram excluídos, posteriormente, na análise dos dados.

Para a coleta de dados foi utilizado um questionário pré-codificado e pré-testado, que incluiu questões elaboradas pelos pesquisadores e questões pertencentes a outros dois instrumentos: Formulário de Marcadores de Consumo Alimentar, constante no protocolo do Sistema de Vigilância Alimentar e Nutricional (SISVAN) 21 e Escala Brasileira de Insegurança Alimentar (EBIA) 22. As informações foram obtidas com as mães/responsáveis pelos escolares.

O desfecho foi excesso de peso, avaliado pelo índice de massa corporal para idade (IMC/I) [peso $(\mathrm{kg})$ / estatura $\left.(\mathrm{m})^{2}\right]$. Definiu-se como excesso de peso um z escore para IMC/I > 121,23,24. Os dados de massa corporal e estatura foram coletados pela Equipe de Nutrição da Secretaria Municipal de Educação, nas dependências das escolas, concomitantemente à realização das entrevistas. As medidas foram obtidas mediante procedimento padrão 25 . A massa corporal foi obtida com balança portátil da marca Plena (São Paulo, Brasil), com capacidade de $150 \mathrm{~kg}$ e precisão de $100 \mathrm{~g}$. Para a estatura foi utilizado estadiômetro portátil da marca Seca (Seca Brasil, Cotia, Brasil), com capacidade de $2 \mathrm{~m}$ e variação de $0,1 \mathrm{~cm}$. 
A variável de exposição foi insegurança alimentar, avaliada pela EBIA. A EBIA foi adaptada e validada no Brasil em 2004 22. A escala consiste em 15 perguntas fechadas, com respostas positivas e negativas, relativas à situação alimentar vivida no domicílio, nos três meses anteriores à entrevista. Para as respostas positivas, foi atribuído o valor 1 (um) e, para as negativas, o valor 0 (zero), resultando num escore com amplitude de 0-15 pontos. A soma dos escores resultantes foi classificada em quatro níveis: 0 (zero) - segurança alimentar; 1-5 - insegurança alimentar leve; 6-10 - insegurança alimentar moderada; e 11-15 - insegurança alimentar grave. Posteriormente, a variável foi recategorizada em segurança alimentar, quando a soma foi igual a 0 (zero), e insegurança alimentar, quando a soma foi $\geq 1$.

As variáveis independentes foram: sexo do escolar (feminino/masculino); idade da mãe/ responsável (coletada em anos completos e categorizada em faixas etárias de 10 anos); cor da pele da mãe/responsável (autorreferida e categorizada em branca e não branca); peso ao nascer do escolar (coletado em gramas, com base na informação referida pela mãe/responsável, confirmado na caderneta da criança, e classificado em < 2.500g; 2.500g-3.999g e $\geq 4.000 \mathrm{~g}$ ); aleitamento materno (coletado com as perguntas: "o escolar mamou no peito? e que idade o escolar tinha quando deixou de mamar"; com base nesta informação, calculou-se o número de dias de amamentação e a variável categorizada em tercis); nível econômico [definido com base no Critério de Classificação Econômica Brasil da Associação Brasileira de Empresas de Pesquisa (ABEP) 26: nível A (35-46 pontos); nível B (23-34 pontos); nível C (14-22 pontos); nível D (8-13 pontos) e nível E (0-7 pontos)]; escolaridade da mãe/responsável (coletada em anos completos de estudos e categorizada em: menos de 4 , de 4-8, e mais de 8 anos); atividade física (coletada com a pergunta: "em quantos dias da última semana o escolar realizou atividades como correr, pular corda, andar de bicicleta, jogar futebol etc, que fizeram com que ele(a) suasse muito ou respirasse mais forte do que o normal" 27, e categorizada em ativo e insuficientemente ativo, sendo considerado ativo o escolar que realizou atividades todos os dias). A utilização dessa forma de obter o dado considerou que questionários para medir o nível de atividade em crianças na faixa etária do presente estudo são escassos e apresentam pobre validade 28 . Além disso, crianças menores apresentam menos probabilidade de participarem em atividades estruturadas 29 . Horas em atividades sedentárias (obtidas como o somatório do número de horas que o escolar ficou em frente à televisão, jogando videogame ou no computador, em um dia típico, e categorizado em: $\leq 2$ horas e $>2$ horas) 27 ; a ingestão alimentar foi estabelecida com base na frequência de ingestão de marcadores de alimentação saudável e não saudável. Para investigar os itens alimentares, utilizou-se uma adaptação do Formulário de Marcadores de Consumo Alimentar, constante no protocolo do SISVAN 21. Nesse formulário, os marcadores são apresentados em grupos alimentares. No presente trabalho, optouse por desmembrar esses grupos em alimentos individuais, tendo em vista as dificuldades das mães/responsáveis em responder sobre alimentos agregados, conforme identificado no estudo piloto. Assim, salada crua e legumes e verduras cozidas, por exemplo, foram desmembrados em: alface, repolho, tomate, pepino, couve, moranga, chuchu, cenoura e beterraba. Além dessas modificações, foram incluídos alimentos como arroz, milho, aipim, batata, massa, pães, queijo, carne, frango, peixe, ovo, margarina, manteiga e suco em pó. A inclusão desses alimentos foi feita com base em estudo de padrão alimentar conduzido com mulheres adultas de São Leopoldo. O padrão alimentar dessas mulheres foi utilizado como um indicativo dos alimentos que poderiam fazer parte da dieta dos escolares 30 . Para cada alimento, perguntou-se o número de dias, dos últimos sete dias anteriores à entrevista, que o mesmo foi ingerido. Valendo-se dessa informação, construiu-se um escore alimentar baseado no proposto por Mondini et al. ${ }^{31}$. Os alimentos receberam uma pontuação segundo o número de dias de ingestão. Para os marcadores saudáveis, a pontuação foi: zero ponto - 0-1 dia; 0,25 ponto -2 -3 dias; 0,75 ponto $-4-5$ dias; e 1 ponto - 6-7 dias. Para os marcadores não saudáveis, a pontuação foi inversa. Desse modo, se o escolar referisse ter consumido frutas e biscoito recheado nos últimos 7 dias e legumes nos últimos 5 dias, receberia, respectivamente, 1 , zero e 0,75 ponto, totalizando 1,75 ponto. O somatório gerou um escore que poderia variar de zero a 44 pontos. Esse escore foi categorizado em terços: 1o terço - ingestão menos saudável; 2 o terço ingestão moderadamente saudável; e 3 o terço ingestão mais saudável.

Para a coleta e digitação dos dados foram selecionados e treinados alunos de graduação de cursos da área da saúde. Com o objetivo de avaliar o questionário, bem como o desempenho dos entrevistadores, conduziu-se um estudo piloto com escolares matriculados no 2 o ano do ensino fundamental de uma das escolas municipais.

O controle de qualidade do trabalho de campo foi feito por telefone, em uma amostra aleatória de $10 \%$ dos indivíduos participantes do 
estudo. Aplicou-se um questionário curto, semelhante ao do estudo, incluindo variáveis que não sofriam alteração em curto espaço de tempo.

Os dados foram digitados em dupla entrada, utilizando-se o programa EpiData, versão 3.1 (Epidata Assoc., Odense, Dinamarca), com o objetivo de identificar erros de digitação. Para avaliar os dados antropométricos utilizou-se o programa WHO Anthro 2007 (Organização Mundial da Saúde; http://www.who.int/childgrowth/software/en/). As análises estatísticas foram realizadas nos programas Stata, versão 9.0 (Stata Corp., College Station, Estados Unidos), e IBM SPSS versão 19.0 (IBM Corp., Armonk, Estados Unidos).

As associações da exposição insegurança alimentar e das demais variáveis explanatórias com o desfecho excesso de peso foram testadas por meio do teste qui-quadrado de Pearson e de associação linear. Para fornecer uma estimativa das razões de prevalências (RP) brutas e ajustadas, além de seus respectivos intervalos de 95\% de confiança (IC95\%), utilizou-se regressão de Poisson com variância robusta 32 . Potenciais fatores de confusão (estar associado com exposição e desfecho a um nível de significância de $\mathrm{p}<0,20$ ) foram incluídos na análise multivariável, que foi realizada mediante três modelos de ajuste, seguindo um modelo de determinação do excesso de peso.

Por se tratar de pesquisa envolvendo seres humanos, foram observadas as regras previstas na Resolução $n^{\circ} 196 / 96$, e o protocolo de pesquisa foi submetido ao Comitê de Ética em Pesquisa da Universidade do Vale do Rio dos Sinos (UNISINOS) e aprovado sob o número 11/013. A mãe/responsável pelo escolar só respondia à entrevista após a leitura e assinatura do Termo de Consentimento Livre e Esclarecido.

\section{Resultados}

Foram realizadas 847 entrevistas, sendo 53,9\% delas conduzidas nos domicílios. Em duas das 35 escolas não foi possível realizar a pesquisa por questões de segurança, uma vez que as mesmas estavam localizadas em áreas com alto índice de criminalidade. Para as análises foram excluídos 16 escolares que estavam realizando dietas especiais, 18 que tinham mais de $30 \%$ de dados faltantes no questionário de consumo alimentar, e 31 por não terem realizado avaliação antropométrica, totalizando 782 indivíduos. Com esse tamanho de amostra foi possível estimar a prevalência de excesso de peso com erro de 3,5 pontos percentuais. Para as associações, esta amostra teve poder de $80 \%$ e nível de $95 \%$ de con- fiança para detectar razões de prevalência de, no mínimo, 1,3.

Nesta amostra, 45,1\% das famílias apresentavam algum grau de insegurança alimentar e $38,1 \%$ dos escolares apresentavam excesso de peso (Tabela 1).

Dentre os 782 escolares avaliados, 52,9\% eram do sexo masculino, a idade média foi de $6,9 \pm 0,6$ anos, a maioria $(80,6 \%)$ apresentou peso de nascimento adequado e apenas $10,4 \%$ não recebeu nenhum tipo de aleitamento materno. Em relação ao tempo em atividades sedentárias, identificou-se que $83,1 \%$ dos escolares passavam 2 horas ou mais por dia na frente da televisão, do computador ou jogando videogame, e menos da metade da amostra $(40,9 \%)$ referiu realizar atividades nos sete dias anteriores à entrevista. Quanto às características demográficas e socioeconômicas das famílias dos escolares, verificou-se que 40,4\% das mães/responsáveis tinham entre 30 e 39 anos, $77,2 \%$ referiram ter cor da pele branca e $53,7 \%$ tinham entre 4 e 8 anos de estudos. A maioria das famílias encontrava-se na classe econômica $\mathrm{D}(59,2 \%)$ e nenhuma família pertencia à classe A (Tabela 1).

A Tabela 2 apresenta as razões de prevalência brutas para excesso de peso de acordo com as características da amostra. Observa-se que a probabilidade de excesso de peso foi maior em crianças cujo peso ao nascer foi $>4.000 \mathrm{~g}$; comparadas às que nasceram com peso entre $2.500 \mathrm{~g}$ e 3.999g, foi diretamente associada à idade da mãe/responsável e inversamente associada ao nível econômico. Na análise bruta, escolares com insegurança apresentaram probabilidade $27 \%$ menor de ter excesso de peso quando comparados àqueles sem insegurança alimentar.

Na análise ajustada, a condição de insegurança alimentar permaneceu inversamente associada ao excesso de peso, independentemente do modelo utilizado, com pequena variação na sua magnitude, de $27 \%$ para $22 \%$ (Tabela 3 ).

\section{Discussão}

Este trabalho teve como objetivo investigar a associação entre insegurança alimentar e excesso de peso entre escolares do 1o ano do Ensino Fundamental da rede municipal de ensino de São Leopoldo. Nesta amostra, as prevalências de excesso de peso e insegurança alimentar foram elevadas, respectivamente, $38,1 \%$ e $45,1 \%$, e a probabilidade de excesso de peso foi $22 \%$ menor nos escolares cujas famílias apresentavam insegurança alimentar, após ajuste para potenciais fatores de confusão. 
Tabela 1

Distribuição da amostra de acordo com as variáveis da família e dos escolares. São Leopoldo, Rio Grande do Sul, Brasil, 2011 $(n=782)$.

\begin{tabular}{|c|c|c|}
\hline Variáveis & $\mathbf{n}$ & $\%$ \\
\hline \multicolumn{3}{|l|}{ Idade da mãe/responsável (anos) } \\
\hline $20-29$ & 288 & 36,8 \\
\hline $30-39$ & 316 & 40,4 \\
\hline$\geq 40$ & 178 & 22,8 \\
\hline \multicolumn{3}{|l|}{ Cor da pele da mãe/responsável } \\
\hline Branca & 604 & 77,2 \\
\hline Não branca & 177 & 22,6 \\
\hline \multicolumn{3}{|l|}{ Escolaridade da mãe/responsável (anos) } \\
\hline$>8$ & 270 & 34,4 \\
\hline $4-8$ & 421 & 53,7 \\
\hline$<4$ & 91 & 11,6 \\
\hline \multicolumn{3}{|l|}{ Nível econômico } \\
\hline Classe $\mathrm{E}$ & 181 & 23,1 \\
\hline Classe D & 463 & 59,3 \\
\hline Classes B e C & 136 & 17,4 \\
\hline \multicolumn{3}{|l|}{ Insegurança alimentar } \\
\hline Segurança alimentar & 429 & 54,9 \\
\hline Insegurança alimentar leve & 269 & 34,4 \\
\hline Insegurança alimentar moderada & 56 & 7,1 \\
\hline Insegurança alimentar grave & 28 & 3,6 \\
\hline \multicolumn{3}{|l|}{ Sexo do escolar } \\
\hline Masculino & 414 & 52,9 \\
\hline Feminino & 368 & 47,1 \\
\hline \multicolumn{3}{|l|}{ Peso ao nascimento } \\
\hline $2.500 \mathrm{~g}-3.999 \mathrm{~g}$ & 630 & 80,6 \\
\hline$<2.500 \mathrm{~g}$ & 87 & 11,1 \\
\hline$>4.000 \mathrm{~g}$ & 65 & 8,3 \\
\hline \multicolumn{3}{|l|}{ Aleitamento materno (dias) } \\
\hline$>730$ & 245 & 31,3 \\
\hline $180-730$ & 276 & 35,3 \\
\hline$<180$ & 261 & 33,4 \\
\hline \multicolumn{3}{|l|}{ Escore alimentar * } \\
\hline Mais saudável & 252 & 32,2 \\
\hline Moderadamente saudável & 240 & 30,7 \\
\hline Menos saudável & 290 & 37,1 \\
\hline \multicolumn{3}{|l|}{ Comportamento sedentário (horas) } \\
\hline$\leq 2$ & 132 & 16,9 \\
\hline$>2$ & 650 & 83,1 \\
\hline \multicolumn{3}{|l|}{ Atividade física } \\
\hline Ativo & 320 & 40,9 \\
\hline Insuficientemente ativo & 462 & 59,1 \\
\hline \multicolumn{3}{|l|}{ Excesso de peso } \\
\hline Sim & 298 & 38,1 \\
\hline Não & 484 & 61,9 \\
\hline
\end{tabular}

IC95\%: intervalo de 95\% de confiança.

* Menos saudável: 1ㅇ terço - 14,0-21,5 pontos; moderadamente saudável: 2o terço - 21,6-23,2 pontos; mais saudável: 3ㅇ terço $-23,3-32,0$ pontos. 
Tabela 2

Razões de prevalência (RP) de excesso de peso de acordo com as variáveis da família e dos escolares. São Leopoldo, Rio Grande do Sul, Brasil, 2011 ( $n=782$ ).

\begin{tabular}{|c|c|c|c|}
\hline Variáveis & RP & IC95\% & Valor de $p$ \\
\hline Idade da mãe/responsável (anos) & & & 0,067 \\
\hline $20-29$ & 1,00 & & \\
\hline $30-39$ & 1,02 & $0,77-1,34$ & \\
\hline$\geq 40$ & 1,30 & $1,01-1,67$ & \\
\hline Cor da pele da mãe/responsável & & & 0,676 \\
\hline Branca & 1,00 & & \\
\hline Não branca & 1,05 & $0,82-1,35$ & \\
\hline Escolaridade da mãe/responsável (anos) & & & 0,210 \\
\hline$>8$ & 1,00 & & \\
\hline $4-8$ & 0,94 & $0,75-1,16$ & \\
\hline$<4$ & 0,82 & $0,59-1,13$ & \\
\hline Nível econômico & & & 0,015 \\
\hline Classe $\mathrm{E}$ & 1,00 & & \\
\hline Classe D & 1,13 & $0,92-1,37$ & \\
\hline Classes B e C & 1,51 & $1,01-2,07$ & \\
\hline Insegurança alimentar & & & $<0,001$ \\
\hline Não & 1,00 & & \\
\hline Sim & 0,73 & $0,62-0,88$ & \\
\hline Sexo do escolar & & & 0,761 \\
\hline Masculino & 1,00 & & \\
\hline Feminino & 1,02 & $0,87-1,20$ & \\
\hline Peso ao nascimento & & & 0,002 \\
\hline $2.500 g-3.999 g$ & 1,00 & & \\
\hline$<2.500 \mathrm{~g}$ & 0,91 & $0,72-1,13$ & \\
\hline$>4.000 \mathrm{~g}$ & 1,55 & $1,23-1,94$ & \\
\hline Aleitamento materno (dias) & & & 0,945 \\
\hline$>730$ & 1,00 & & \\
\hline $180-730$ & 0,92 & $0,69-1,21$ & \\
\hline$<180$ & 1,01 & $0,77-1,32$ & \\
\hline Escore alimentar * & & & 0,150 \\
\hline Mais saudável & 1,00 & & \\
\hline Moderadamente saudável & 1,02 & $0,83-1,26$ & \\
\hline Menos saudável & 0,80 & $0,58-1,09$ & \\
\hline Comportamento sedentário (horas) & & & 0,888 \\
\hline$\leq 2$ & 1,00 & & \\
\hline$>2$ & 0,98 & $0,77-1,25$ & \\
\hline Atividade física & & & 0,216 \\
\hline $\operatorname{Sim}$ & 1,00 & & \\
\hline Não & 1,12 & $0,93-1,34$ & \\
\hline
\end{tabular}

IC95\%: intervalo de 95\% de confiança.

* Menos saudável: 1ํ terço - 14,0-21,5 pontos; moderadamente saudável: 2o terço - 21,6-23,2 pontos; mais saudável: 3o terço-23,3-32,0 pontos.

A frequência de excesso de peso no presente estudo é consistente com dados nacionais, de base populacional, como os da $\mathrm{POF} 4$ realizada no Brasil em 2008-2009, que revelou 34\% de ex- cesso de peso entre as crianças de cinco a nove anos. Outros estudos, de base escolar, realizados no país, também encontraram prevalências elevadas, variando de $29,8 \% 33$ a $34,5 \%$. Da mesma 
Tabela 3

Razões de prevalência entre insegurança alimentar e excesso de peso de acordo com os diferentes modelos de ajuste.

\begin{tabular}{|c|c|c|c|c|}
\hline Variáveis & $\begin{array}{c}\text { Modelo I } \\
\text { RP (IC95\%) }\end{array}$ & $\begin{array}{l}\text { Modelo II } \\
\text { RP (IC95\%) }\end{array}$ & $\begin{array}{l}\text { Modelo III } \\
\text { RP (IC95\%) }\end{array}$ & $\begin{array}{l}\text { Modelo IV RP } \\
\text { (IC95\%) }\end{array}$ \\
\hline \multicolumn{5}{|l|}{ Insegurança alimentar } \\
\hline Não & 1,00 & 1,00 & 1,00 & 1,00 \\
\hline Sim & $0,73(0,62-0,88)$ & $0,73(0,61-0,88)$ & $0,78(0,63-0,95)$ & $0,78(0,64-0,96)$ \\
\hline \multicolumn{5}{|c|}{ Idade da mãe/responsável (anos) * } \\
\hline $20-29$ & & 1,00 & 1,00 & 1,00 \\
\hline $30-39$ & & $1,01(0,76-1,34)$ & $0,99(0,77-1,31)$ & $0,98(0,74-1,30)$ \\
\hline$\geq 40$ & & $1,30(1,02-1,66)$ & $1,28(1,00-1,65)$ & $1,28(0,99-1,66)$ \\
\hline \multicolumn{5}{|l|}{ Nível econômico * } \\
\hline Classes B e C & & & 1,00 & 1,00 \\
\hline Classe D & & & $1,04(0,84-1,28)$ & $1,03(0,82-1,28)$ \\
\hline Classe E & & & $1,29(0,92-1,81)$ & $0,79(0,90-1,77)$ \\
\hline \multicolumn{5}{|l|}{ Consumo alimentar * } \\
\hline Mais saudável & & & & 1,00 \\
\hline Moderadamente saudável & & & & $1,05(0,84-1,31)$ \\
\hline Menos saudável & & & & $0,85(0,61-1,18)$ \\
\hline
\end{tabular}

IC95\%: intervalo de 95\% de confiança.

Modelo I: efeito da insegurança alimentar sem ajuste. Modelo II: efeito da insegurança alimentar ajustada para a variável idade da mãe/responsável. Modelo III: efeito da insegurança alimentar ajustada para o Modelo II e nível econômico da família. Modelo IV: efeito da insegurança alimentar ajustada para o Modelo III e hábito alimentar do escolar.

* Variáveis associadas com o desfecho e com a exposição, considerando um nível de significância menor que $20 \%$.

forma, a literatura internacional identificou prevalência semelhante $(31,6 \%)$ entre crianças da mesma faixa etária 34 , confirmando a magnitude do problema.

Quanto à frequência de insegurança alimentar, o resultado do presente trabalho é superior ao encontrado na Pesquisa Nacional por Amostra de Domicílios de 2009 (PNAD 2009), que registrou $30,2 \%$ das famílias brasileiras com algum grau de insegurança alimentar e maiores prevalências nos domicílios com menor renda 19. O elevado número de famílias com insegurança alimentar, em nosso estudo, poderia ser explicado pelas características econômicas da amostra estudada, uma vez que $82,3 \%$ dos escolares pertenciam aos estratos econômicos D e E. Estudo de base populacional, conduzido no distrito mais pobre do Município de Duque de Caxias, Rio de Janeiro, também encontrou alta prevalência de insegurança alimentar (72\%) 35 .

Nosso estudo identificou associação inversa e significativa da insegurança alimentar com excesso de peso, mesmo após ajuste para as variáveis demográficas, socioeconômicas e comportamentais. Entre escolares cujas famílias apresentaram insegurança alimentar, a probabilidade de excesso de peso foi $22 \%$ menor quando comparados àqueles cujas famílias não tinham insegurança alimentar. Esses achados são consistentes com os descritos na literatura 17,18. No Brasil, o estudo realizado em Duque de Caxias, por Pimentel et al. 35, demonstrou que, entre as crianças, a insegurança alimentar apresentou uma relação significativamente linear e negativa com o escore-Z de peso/idade (P/I) e de peso/ estatura (P/E).

Em outros estudos, contudo, os achados apontam para uma associação positiva entre insegurança alimentar e obesidade 11,12. Já os dados da Pesquisa Nacional de Demografia e Saúde de 2006 (PNDS 2006) revelaram que, enquanto insegurança alimentar grave esteve associada com obesidade, entre mulheres adultas $(\mathrm{RP}=1,49$; IC95\%: 1,17-1,90), e insegurança alimentar moderada com excesso de peso entre adolescentes do sexo feminino ( $R P=1,96$; IC95\%: 1,18-3,27), não se observou associação entre insegurança alimentar e obesidade em crianças de ambos os sexos 16. Baseados nos dados da pesquisa National Health and Examination Survey (NHANES) de 2001 e 2004, Gundersen et al. 14 mostraram que insegurança alimentar não esteve associada com nenhuma das medidas de obesidade considerada no estudo, mesmo após controlar para potenciais fatores de confusão. 
Segundo alguns autores 36,37, a associação entre insegurança alimentar e obesidade seria consequência de um processo adaptativo à escassez de alimentos, no qual, por mecanismos fisiológicos, ocorreria poupança de energia e armazenamento de gordura com mais facilidade. Outras hipóteses discutidas na literatura evidenciam que famílias com recursos limitados tendem a comprar alimentos com alta densidade energética e com elevado teor de gordura 36 , e que indivíduos que apresentam acesso limitado tendem a um consumo excessivo quando os alimentos tornam-se disponíveis, como um mecanismo compensatório aos períodos de escassez 37,38 . No presente trabalho, ingestão alimentar menos saudável foi mais prevalente entre escolares com insegurança alimentar, entretanto, não se observou associação desta variável com excesso de peso, mesmo após ajuste. A ingestão alimentar também não se comportou como um mediador da insegurança alimentar para o excesso de peso, uma vez que o ajuste para esta variável não diminuiu a magnitude da medida de efeito. Dados de outro estudo com a mesma população ${ }^{39}$ mostraram que os escores mais altos de consumo alimentar foram compostos tanto por consumo menos frequente dos marcadores de alimentação não saudável, quanto dos marcadores de alimentação saudável, o que poderia explicar a não associação com excesso de peso. Entretanto, é importante ressaltar que para os dados de consumo alimentar foram coletados apenas a frequência, impossibilitando a quantificação do valor energético. A forma como a informação sobre o consumo foi obtida também deve ser considerada. Assim, com a utilização de um respondente proxy não se pode descartar a possibilidade de erro de informação.

Um aspecto importante a ser considerado é que as perguntas da EBIA se referem aos três meses anteriores à entrevista, portanto, não é possível identificar se o grau de insegurança alimentar encontrado reflete uma situação aguda ou crônica. No caso de ser uma situação aguda, talvez ela não fosse suficiente para provocar a adaptação fisiológica e consequente aumento de peso 40 . Por outro lado, se o grau de insegurança alimentar encontrado é uma situação crônica, não se pode descartar a possibilidade de a mãe sacrificar seu consumo de alimentos em prol de seus filhos. Assim, por mais que a família se encontre em algum grau de insegurança alimentar por longo período, a criança não apresentaria as respostas fisiológicas adaptativas à falta de alimentos ${ }^{41}$. Outro aspecto que poderia minimizar a resposta adaptativa é que, por serem escolares, essas crianças têm acesso à alimentação fornecida pela escola 42 . Além disso, os requerimentos energéticos na infância são maiores, situação que pode proteger as crianças contra as influências negativas da insegurança alimentar sobre a obesidade 34 . Contudo, o percentual de crianças com déficit de IMC para a idade em nosso trabalho foi de apenas $1,5 \%$.

Para Schlüssel et al. 16, a falta de associação entre insegurança alimentar e obesidade observada em crianças sugere que tal situação assemelha-se à transição nutricional, conforme descrito por Monteiro et al. ${ }^{43}$, em que a obesidade começa entre os adultos, a seguir acomete os adolescentes e, só mais tarde, afeta as crianças. Assim, o possível efeito da insegurança alimentar sobre a obesidade, em crianças, constituiria uma característica de um estágio muito avançado dessa transição, que o Brasil ainda não atingiu. Conforme revisado por Franklin et al. 41, embora a associação entre insegurança alimentar e obesidade em crianças permanece para ser esclarecida, a mesma já está bem estabelecida entre as mulheres e adolescentes.

Os resultados do presente estudo devem ser discutidos à luz de algumas limitações. Inicialmente, as dificuldades logísticas e financeiras permitiram investigar somente 847 escolares matriculados no 1o ano do Ensino Fundamental das escolas municipais. Entretanto, a frequência de excesso de peso entre os escolares que não participaram do estudo (39,7\%; IC95\%: $37,2 \%-42,3 \%$ ) foi semelhante à dos participantes (38,1\%; IC95\%: 34,7\%-41,5\%), sugerindo menor probabilidade de viés de seleção. Além disso, a amostra estudada incluiu, proporcionalmente, escolares de todas as escolas, com exceção das duas mencionadas anteriormente, o que contemplou as diferentes regiões do município. Como o presente trabalho investigou apenas escolares da rede municipal, se desconhece como a relação entre insegurança alimentar e excesso de peso acontece em escolares das redes estadual e privada de ensino. A forma como a variável de exposição insegurança alimentar foi categorizada também representa uma limitação, pois foram considerados numa única categoria todos os níveis de insegurança alimentar e sabe-se que cada nível representa diferentes situações, desde a preocupação com a obtenção do alimento (leve), passando pela redução da qualidade e variedade de alimentos (moderada) até a falta do alimento (grave) 44, e estas poderiam se relacionar diferentemente com o excesso de peso. A opção por agrupar todos os níveis numa única categoria se deu em função do pequeno número de domicílios com insegurança alimentar moderada e grave, o que resultou em perda de poder estatístico, embora as associações tenham sido sempre na mesma direção daquela obtida com a insegurança alimentar agrupada. A impossibili- 
dade de avaliar a influência do estado nutricional materno sobre o estado nutricional do escolar foi considerada outra limitação do estudo, já que esta informação não foi coletada. O delineamento transversal também é uma limitação do presente trabalho, visto que não é possível estabelecer temporalidade entre a exposição e o desfecho avaliados 45 .

Neste estudo, observou-se relação inversa entre insegurança alimentar e excesso de peso, ambas as condições foram altamente prevalentes, especialmente entre os escolares do pior estrato socioeconômico (E): $74 \%$ e $32,6 \%$ para insegurança alimentar e excesso de peso, res-

\section{Resumen}

Estudio transversal para evaluar la asociación entre la inseguridad alimentaria y el sobrepeso en niños matriculados en el primer año de la enseñanza primaria en las escuelas municipales de São Leopoldo, Rio Grande do Sul, Brasil. Se invitó a 2.369 estudiantes, 847 fueron investigados y de éstos, 782 tenían datos sobre el peso y la altura. Los datos se obtuvieron de los padres/tutores. La inseguridad alimentaria se midió a través de la Escala Brasileña de Inseguridad Alimentaria (EBIA). Peso y altura se obtuvieron con el Equipo de Nutrición de la Educación Municipal. Las prevalencias de sobrepeso y inseguridad alimentaria fueron, respectivamente, $38,1 \%$ y 45,1\%. Después de ajustar por factores de confusión, los niños con inseguridad alimentaria tenían una probabilidad $22 \%$ menor de tener sobrepeso, en comparación con los que no tienen inseguridad alimentaria. Este estudio identificó una alta prevalencia de la inseguridad alimentaria y sobrepeso, con una asociación inversa entre estas variables. Por un lado, estos resultados ponen de manifiesto la complejidad de esta relación, lo que requiere más estudios para entender, $y$ por otro, demuestra la necesidad de políticas públicas sólidas para hacer frente a estas condiciones.

Obesidad Pediátrica; Sobrepeso; Estudiantes; Seguridad Alimentaria pectivamente. Além disso, consumo alimentar menos saudável foi mais frequente entre os escolares que apresentaram insegurança alimentar. Esses achados reiteram a necessidade de políticas públicas robustas e distributivas, que garantam acesso a alimentos de qualidade, em quantidade suficiente, e educação alimentar para que essa oferta possa promover saúde e bem-estar. Quanto à associação entre insegurança alimentar e excesso de peso, há necessidade de estudos prospectivos, os quais permitem estabelecer uma relação causal, pois atendem ao critério da temporalidade.

\section{Colaboradores}

K. Vicenzi participou da coordenação da análise, interpretação e discussão dos resultados, redação e revisão do artigo. R. L. Henn coordenou a análise, interpretação e discussão dos resultados, redação e revisão do artigo. A. P.Weber, V. Backes, T. Donatti participou da discussão dos resultados e revisão do artigo. V. M. V. Paniz colaborou na análise e discussão dos resultados e na revisão do artigo. M. T. A. Olinto colaborou no planejamento do estudo e na revisão do artigo.

\section{Agradecimentos}

Os autores agradecem aos entrevistadores pela coleta dos dados e à Equipe de Nutrição da Secretaria Municipal de Educação de São Leopoldo, pelos dados antropométricos. 


\section{Referências}

1. Ng M, Fleming T, Robinson M, Thomson B, Graetz N, Margono C, et al. Global, regional, and national prevalence of overweight and obesity in children and adults during 1980-2013: a systematic analysis for the Global Burden of Disease Study 2013. Lancet 2014; 384:766-81.

2. World Health Organization. Obesity: preventing and managing the global epidemic. Report of WHO Consultation in Obesity. Geneva: World Health Organization; 2000.

3. de Onis M, Blossner M, Borghi E. Global prevalence and trends of overweight and obesity among preschool children. Am J Clin Nutr 2010; 92: 1257-64.

4. Instituto Brasileiro de Geografia e Estatística. Pesquisa de Orçamentos Familiares 2008-2009. Antropometria e análise do estado nutricional de crianças, adolescentes e adultos no Brasil. Rio de Janeiro: Instituto Brasileiro de Geografia e Estatística; 2010.

5. Barker DJ. The developmental origins of chronic adult disease. Acta Paediatr Suppl 2004; 93:26-33.

6. Rossi CE, Vasconcelos FAG. Relationship between birth weight and overweight/obesity among students in Florianópolis, Santa Catarina, Brazil: a retrospective cohort study. São Paulo Med J 2014 132:273-81.

7. Oddy WH, Mori TA, Huang RC, Marsh JA, Pennell CE, Chivers PT, et al. Early infant feeding and adiposity risk: from infancy to adulthood. Ann Nutr Metab 2014; 64:262-70.

8. Bernardo CO, Pudla KJ, Longo GZ, Vasconcelos FA. Fatores associados ao estado nutricional de escolares de 7 a 10 anos: aspectos sociodemográficos, de consumo alimentar e estado nutricional dos pais. Rev Bras Epidemiol 2012; 15:651-61.

9. Pradinuk M, Chanoine JP, Goldman RD. Obesity and physical activity in children. Can Fam Physician 2011; 57:779-82.

10. Brasil. Lei no 11.346 , de 15 de setembro de 2006 . Cria o Sistema Nacional de Segurança Alimentar e Nutricional - SISAN com vistas em assegurar o direito humano à alimentação adequada e dá ou tras providências. http://www4.planalto.gov.br/ consea/conferencia/documentos/lei-de-segu ranca-alimentar-e-nutricional (acessado em 30/ Out/2014).

11. Casey PH, Simpson PM, Gossett JM, Bogle ML, Champagne CM, Connell C, et al. The association of child and household food insecurity with childhood overweight status. Pediatrics 2006; 118:e1406-13.

12. Dubois L, Francis D, Burnier D, Tatone-Tokuda F, Girard M, Gordon-Strachan G, et al. Household food insecurity and childhood overweight in Jamaica and Quebec: a gender-based analysis. BMC Public Health 2011;11:199.

13. Kaiser LL, Lamp CL, John MC, Sutherlin JM, Harwood JO. Food security and nutritional outcomes of preschool-age Mexican-American children. J Am Diet Assoc 2002; 102:924-9.
14. Gundersen C, Garasky S, Lohman BJ. Food insecurity is not associated with childhood obesity as assessed using multiple measures of obesity. J Nutr 2009; 139:1173-8.

15. Martin KS, Ferris AM. Food insecurity and gender are risk factors for obesity. J Nutr Educ Behav 2007; 39:31-6.

16. Schlüssel MM, Silva AAM, Pérez-Escamilla R, Kac G. Household food insecurity and excess weight/ obesity among Brazilian women and children: a life-course approach. Cad Saúde Pública 2013; 29:219-26.

17. Rose D, Bodor JN. Household food insecurity and overweight status in young school children: results from the Early Childhood Longitudinal Study. Pediatrics 2006; 117:464-73.

18. Matheson DM, Varady J, Varady A, Killen JD. Household food security and nutritional status of Hispanic children in the fifth grade. Am J Clin Nutr 2002; 76:210-7.

19. Instituto Brasileiro de Geografia e Estatística. Pesquisa Nacional por Amostra de Domicílios PNAD. Brasília: Instituto de Pesquisa de Geografia e Estatística; 2009.

20. Coordenação-Geral da Política de Alimentação e Nutrição, Departamento de Atenção Básica, Secretaria de Atenção à Saúde, Ministério da Saúde. Relatório de Gestão-2007. http://189.28.128.100/ nutricao/docs/geral/relatorio_2007_cgpan.pdf (acessado em 20/Mar/2015)

21. Departamento de Atenção Básica, Secretaria de Atenção à Saúde, Ministério da Saúde. Protocolos do Sistema de Vigilância Alimentar e Nutricional SISVAN na assistência à saúde. Brasília: Ministério da Saúde; 2008.

22. Segall-Corrêa AM, Pérez-Escamilla R, Maranha LK, Sampaio MFA, Yuyama L, Alencar F, et al. Projeto: acompanhamento e avaliação da segurança alimentar de famílias brasileiras: validação de metodologia e de instrumento de coleta de informação. Campinas: Departamento de Medicina Preventiva e Social, Universidade Estadual de Campinas/Organização Pan-Americana da Saúde/Ministério da Saúde; 2003. (Relatório Técnico).

23. de Onis M, Onyango AW, Borghi E, Siyam A, Nishida C, Siekmann J. Development of a WHO growth reference for school-aged children and adolescents. Bull World Health Organ 2007; 85:660-7.

24. WHO Multicentre Growth Reference Study Group. WHO child growth standards: length/height-forage, weight-for-age, weight-for-length, weight-forheight and body mass index-for-age: methods and development. Geneva: World Health Organization; 2006.

25. World Health Organization. Physical status: the use and interpretation of anthropometry. Report of a WHO Expert Committee. Geneva: World Health Organization; 1995; (Technical Report Series, 854). 
26. Associação Brasileira de Empresas de Pesquisa. Critérios de classificação econômica Brasil 2010. São Paulo: Associação Brasileira de Empresas de Pesquisa; 2010.

27. Anderson SE, Economos CD, Must A. Active play and screen time in US children aged 4 to 11 years in relation to sociodemographic and weight status characteristics: a nationally representative crosssectional analysis. BMC Public Health 2008; 8:366.

28. Telford A, Salmon J, Jolley D, Crawford D. Reliability and validity of physical activity questionnaires for children: the Children's Leisure Activities Study Survey (CLASS). Pediatr Exerc Sci 2004; 16:64-78.

29. Biddle S, Goudas M. Analysis of children's physical activity and its association with adult encouragement and social cognitive variables. J Sch Health 1996; 66:75-9.

30. Alves AL, Olinto MT, Costa JS, Bairros FS, Balbinotti MA. Dietary patterns of adult women living in an urban area of Southern Brazil. Rev Saúde Pública 2006; 40:865-73

31. Mondini L, Levy RB, Saldiva SR, Venâncio SI, Azevedo Aguiar J, Stefanini ML. Prevalência de sobrepeso e fatores associados em crianças ingressantes no ensino fundamental em um município da região metropolitana de São Paulo, Brasil. Cad Saúde Pública 2007; 23:1825-34.

32. Barros AJ, Hirakata VN. Alternatives for logistic regression in cross-sectional studies: an empirical comparison of models that directly estimate the prevalence ratio. BMC Med Res Methodol 2003; $3: 21$.

33. Vieira MFA, Araújo CLP, Hallal PC, Madruga SW, Neutzling MB, Matijasevich A, et al. Estado nutricional de escolares de 1ạ a 4a séries do Ensino Fundamental das escolas urbanas da cidade de Pelotas, Rio Grande do Sul, Brasil. Cad Saúde Pública 2008; 24:1667-74.

34. Singh GK, Kogan MD, van Dyck PC. Changes in state-specific childhood obesity and overweight prevalence in the United States from 2003 to 2007. Arch Pediatr Adolesc Med 2010; 164:598-607.
35. Pimentel PG, Sichieri R, Salles-Costa R. Insegurança alimentar, condições socioeconômicas e indicadores antropométricos em crianças da Região Metropolitana do Rio de Janeiro/Brasil. Rev Bras Estud Popul 2009; 26:283-94.

36. Alaimo K, Olson CM, Frongillo Jr. EA. Low family income and food insufficiency in relation to overweight in US children: is there a paradox? Arch Pediatr Adolesc Med 2001; 155:1161-7.

37. Dietz WH. Critical periods in childhood for the development of obesity. Am J Clin Nutr 1994; 59:955-9.

38. Scheier LM. What is the hunger-obesity paradox? J Am Diet Assoc 2005; 105:883-5.

39. Ruschel LF. Insegurança alimentar e consumo alimentar em escolares do primeiro ano do ensino fundamental da rede municipal de São Leopoldo, RS [Dissertação de Mestrado]. São Leopoldo: Universidade do Vale do Rio dos Sinos; 2014.

40. Dietz WH. Does hunger cause obesity? Pediatrics 1995; 95:766-7.

41. Franklin B, Jones A, Love D, Puckett S, Macklin J, White-Means S. Exploring mediators of food insecurity and obesity: a review of recent literature. J Community Health 2012; 37:253-64.

42. Fundo Nacional de Desenvolvimento da Educação, Secretaria de Educação a Distância, Ministério da Educação. Programa Nacional de Formação Continuada a Distância nas Ações do FNDE. 2aa Ed. 2008. ftp://ftp.fnde.gov.br/web/formacao_pe la_escola/modulo_pnae_conteudo.pdf (acessado em 20/Mar/2015).

43. Monteiro CA, Conde WL, Popkin BM. Is obesity replacing or adding to undernutrition? Evidence from different social classes in Brazil. Public Health Nutr 2002; 5:105-12.

44. Kepple AW, Segall-Corrêa AM. Conceituando e medindo segurança alimentar e nutricional. Ciênc Saúde Coletiva 2011; 16:187-99.

45. Rothman K. Epidemiology: an introduction. Oxford: Oxford University Press; 2002.

Recebido em 07/Abr/2014

Versão final reapresentada em 30/Out/2014 Aprovado em 15/Dez/2014 